\title{
Tables of legislation and decisions
}

\begin{tabular}{|c|c|}
\hline EPC - Articles & 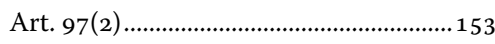 \\
\hline & 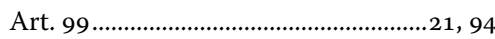 \\
\hline 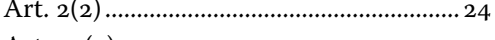 & Art. $99(1) \ldots \ldots \ldots \ldots \ldots \ldots \ldots \ldots \ldots \ldots, 24,55,76,99,124$ \\
\hline Art. 14(4) ......................................... 27, 101 & Art. $100 \ldots \ldots \ldots \ldots . \ldots 21,32,49,62,63,64,161$ \\
\hline Art. $19 \ldots \ldots \ldots \ldots \ldots . . .23$ & Art $100(a)$ \\
\hline Art. $19(2)$ & $.28,32,33-0,37,38-40$ \\
\hline$\ldots \ldots \ldots \ldots \ldots \ldots \ldots \ldots 141$ & $\begin{array}{r}41-6,47 \\
4\end{array}$ \\
\hline ......................... 94 & $\ldots 11,28,32,47-52,63,161$ \\
\hline 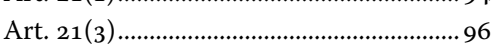 & Art. $100(\mathrm{c}) \ldots \ldots .5,6,8,12,17,18,19,28,32$, \\
\hline 96 & $53-4,69,111$ \\
\hline . & rt. $101 \ldots+21$ \\
\hline 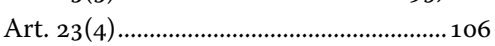 & 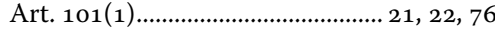 \\
\hline 142 & Art. $101(2) \ldots$. \\
\hline 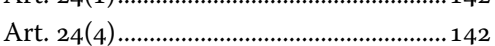 & Art. $101(3) \ldots \ldots \ldots \ldots$ \\
\hline $52 \ldots$. & . \\
\hline $2 \ldots$. & Art. 101(3)(b) \\
\hline Ar & Art. $103 \ldots \ldots \ldots \ldots . . . . .$. \\
\hline .......32, 47 & 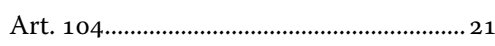 \\
\hline Art. $53(\mathrm{~b}) \ldots$ & 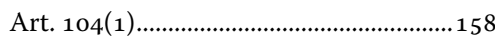 \\
\hline Art. $53(\mathrm{c}) \ldots$. & 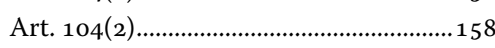 \\
\hline Art. $54 \ldots \ldots \ldots . . .$. & 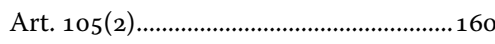 \\
\hline 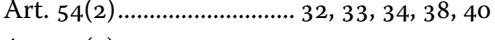 & Art. $106 \ldots \ldots \ldots \ldots \ldots . . . .$. \\
\hline $32,135,136$ & Art. $107 \ldots \ldots \ldots \ldots \ldots \ldots \ldots \ldots . . . . \ldots 6,98,99,132,133$ \\
\hline Art. $55 \ldots \ldots \ldots$. & $\ldots \ldots . . . . .99,100,101,104$ \\
\hline$\ldots .12,32,71,102$ & 155 \\
\hline $57 \cdots \cdots$ & Art. $109(1) \ldots \ldots .$. \\
\hline Art. $61 \ldots \ldots \ldots$ & Art. $109(2) \ldots \ldots .$. \\
\hline 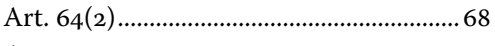 & Art. $111(1) \ldots \ldots . .$. \\
\hline .................., 36 & Art. $111(2) \ldots \ldots .$. \\
\hline ...................... 32 & Art. $112 \ldots \ldots \ldots . .$. \\
\hline 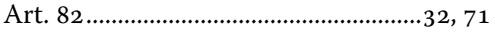 & Art. $112(1) \ldots . .$. \\
\hline$\ldots \ldots . .11,48,49,71,103,137$ & Art. $112(1)(a)$ \\
\hline Art. $84 \ldots \ldots \ldots ., 10,11,17,32,49,63-5,72$, & Art. $112 \mathrm{a} \ldots \ldots \ldots . . . .$. \\
\hline $103,117,148,156$ & Art. $112 \mathrm{a}(1) \ldots . . .$. \\
\hline ................6, 53 & Art. $112 \mathrm{a}(2) \ldots$. \\
\hline$\ldots \ldots \ldots . . . .3,5$ & Art. $112 \mathrm{a}(3) \ldots$ \\
\hline ........... 94 & Art. $112 \mathrm{a}(4) \ldots$. \\
\hline
\end{tabular}


Art. 112a(5)

Art. 113

Art. 113(1)

Art. 113(2)

Art. 114..

Art. 114(1)

Art. 114(2)

Art. 115

Art. 116

Art. 116(1)

Art. 116(3).

Art. $117(1)(d)$

Art. 123

Art. 123(2).

$.5,6,8,12,14,16,17,18$, $19,20,37,53,62,66,69,71,72$, $79,80,84,111,117,123,134,135$, 137,156

Art. 123(3)......14, 16, 17, 18, 19, 62, 65-7, $68,69,71,72,84,135,137$

Art. 133(2) .. 29

Art. 134 .29

\section{EPC - Implementing Regulations}

Rule 3(1)

Rule 12(2) 27,101

Rule 43(2)

Rule 49(8)

Rule 50(3).

Rule 71(3)

Rule 76.

Rule 76(2).

Rule 76(2)(a)

Rule 76(2)(b).

Rule 76(2)(c)

Rule 76(2)(d). ...106 $.70,71$ .. 80

Rule 76(3)....

Rule 77 ....

Rule 77(1).

Rule 77(2).

Rule 79(1)

Rule 80

Rule 82(2).

Rule 82(3)

Rule 84(1)
147

142,143

61,112

56

56,122

92

72

.75

81

16

,

(

Rule 84(2) ................................................ 92

Rule 86........................................................... 92

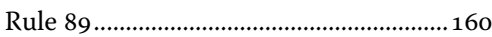

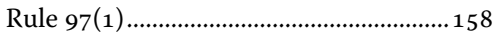

Rule 97(2) ..................................................158

Rule 99(1) ...................................................... 99

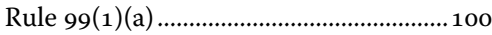

Rule 99(2) ........................................99, 107

Rule 99(3) ...................................................100

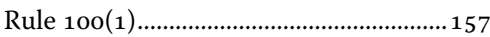

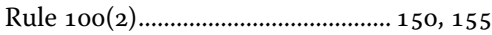

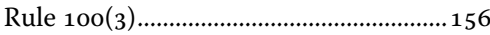

Rule 101(1).................................................101

Rule 101(2).................................................101

Rule 102.......................................................... 139

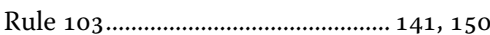

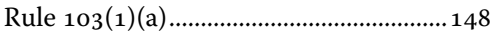

Rule 103(1)(b) ................................ 99, 150

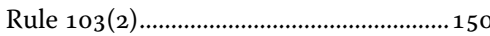

Rule 104............................................ 142, 144

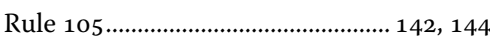

Rule 106.................................142, 143, 144

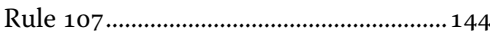

Rule 107(1) ................................................... 144

Rule 107(1)(a)...........................................144

Rule 107(1)(b) ..........................................144

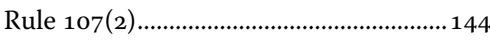

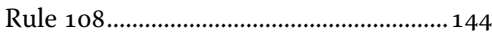

Rule 108(1) .................................................... 144

Rule 108(2)................................................. 144

Rule 108(3).................................................. 147

Rule 109 ..................................................... 147

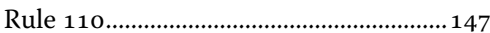

Rule 111(2)...........................................8 87, 88

Rule 114(1)................................................. 92

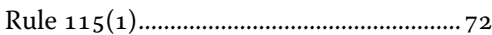

Rule 116(1)..................................59, 72, 73

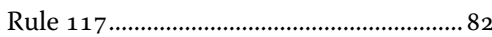

Rule 118(1) .................................................. 82

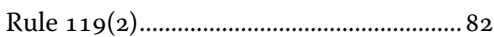

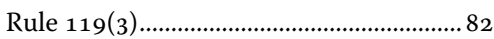

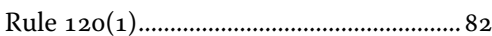

Rule 124(1)................................................... 82

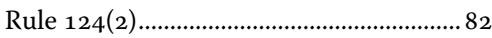

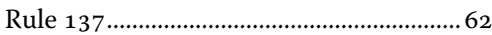


Rule 137(3)...................................................157

Rule 139. $14,15,67,69,70,101$

G 10/93 156

Rule 140 ..69, 70 G $1 / 94$ .161

Rule 152(2) .73

Rule 152(6). .73

Rules of Procedure of the Boards of Appeal (RPBA)

Art. 12(1) 145

Art. 12(2) 106, 107

Art. 12(4) $107,108,110-12,113,116$, $121-3,124,126$

Art. 13 . 120

Art. 13(1)......... 107, 108, 109, 110, 113-14, $115,116,121,124-5,126$ Art. 13(3)............... 107, 108, 109, 110, 113, $115-18,121,124,126,127$

Art. 15(3) 131

Art. 16(1) .159

Art. 16(1)(a) 159

Art. 16(1)(c) .159

Art. 16(1)(e) 159

\section{G Decisions/Opinions}

$\mathrm{G}_{1} / 84$ 122

$\mathrm{G}_{2} / 88$ .68

$\mathrm{G} 4 / 88$ 30

$\mathrm{G} 1 / 91$ 71

$\mathrm{G} 2 / 91$ 95,151

$\mathrm{G} 4 / 91$ 161

G 8/91 96,151

G 9/91 $.31,56,62,64,95,102,104$, 105,161

G 10/91 $31,56,95,105,161$

G 11/91 .... 14

G 1/92 $.38,39$

$\mathrm{G} 4 / 92$ $62,84,128$

G 8/92 139

G 9/92 $96,102,132,133,144$ G 1/93 19,20

$\mathrm{G}$ 7/93 129,141

G 8/93 96,151 G 9/93

$\mathrm{R} 1 / 08$ 145

$\mathrm{R} 2 / 08$ 145

$\mathrm{R}_{4} / 08$ 143 .30 


\begin{tabular}{|c|c|}
\hline$\ldots .143$ & $291 / 85 \ldots \ldots$ \\
\hline$\ldots . . .145$ & $\mathrm{~T} 63 / 86 \ldots$ \\
\hline 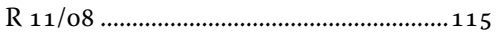 & Т $113 / 86 \ldots . .$. \\
\hline 6/09.............. & Г $234 / 86 \ldots .$. \\
\hline 7/09:.............................. 147, 148 & $\mathrm{~T}_{389} / 86 \ldots \ldots$. \\
\hline 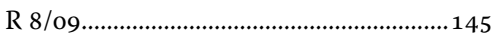 & Т 256/87 \\
\hline 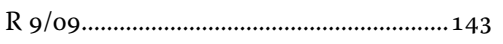 & Т 293/87 \\
\hline 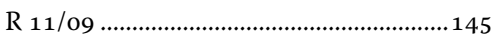 & Т 301/87 \\
\hline 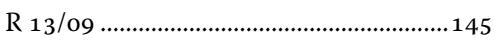 & 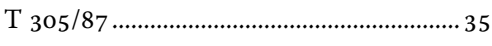 \\
\hline R 16/09 …................................115, 142 & 150 \\
\hline $19 / 09$ & 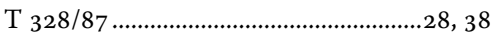 \\
\hline ................. 95, 143 & 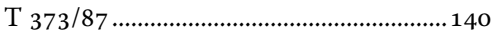 \\
\hline 3/10 & Т 26/88 \\
\hline$\ldots+\ldots \ldots+\ldots \ldots+\ldots 138$ & $\mathrm{~T} 153 / 88 \ldots \ldots$. \\
\hline ..................145 & Т $198 / 88 \ldots . . .$. \\
\hline $17 / 10$ & T 208/88 ...........................................140 \\
\hline 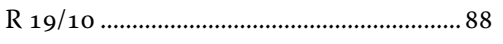 & 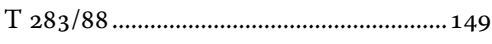 \\
\hline 146 & T $379 / 88 \ldots . . . .$. \\
\hline $15 / 11 \ldots \ldots \ldots \ldots \ldots .146,147,148$ & Т $383 / 88$ \\
\hline 16/11 .................................................146 & Т $461 / 88 \ldots \ldots$. \\
\hline ......................... 145 & Т $536 / 88 \ldots . . .$. \\
\hline 146 & Т $182 / 89 \ldots \ldots$. \\
\hline 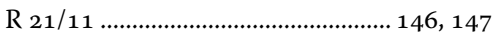 & 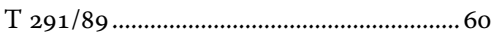 \\
\hline 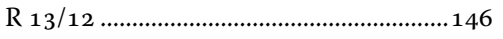 & Т $386 / 89$ \\
\hline 15/12 ….............................................146 & T $454 / 89 \ldots \ldots \ldots \ldots \ldots$ \\
\hline$\ldots \ldots \ldots \ldots \ldots \ldots \ldots \ldots . .143$ & ............................ \\
\hline ................96 & $\mathrm{T} 534 / 89 \ldots$ \\
\hline$\ldots 120$ & 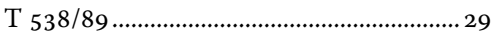 \\
\hline & .......................... 46 \\
\hline & 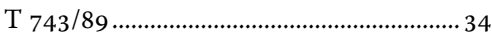 \\
\hline & $\mathrm{T} 774 / 89 \ldots \ldots . .$. \\
\hline .............152 & $\mathrm{T} 5 / 90 \ldots \ldots . . .$. \\
\hline $\mathrm{T}_{32} / 82 \ldots \ldots$. & $\mathrm{T} 34 / 90 \ldots . . . .$. \\
\hline …….................... 45 & T $398 / 90 \ldots . . .$. \\
\hline 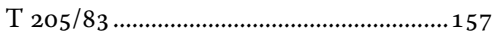 & 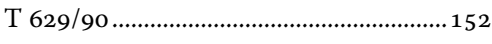 \\
\hline Т 13/84 & 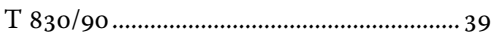 \\
\hline 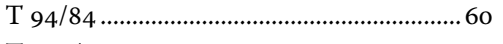 & 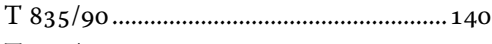 \\
\hline$\ldots+122$ & Т 877/90 \\
\hline$\ldots 141$ & T $125 / 91 \ldots$. \\
\hline 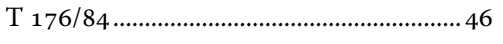 & 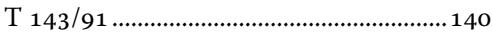 \\
\hline T 194/84 & 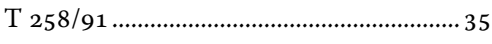 \\
\hline 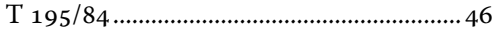 & 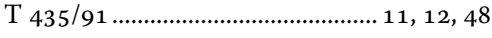 \\
\hline Т 258/84 & T 506/91 …....................................... 95 \\
\hline 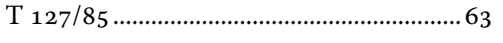 & 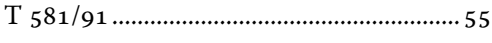 \\
\hline 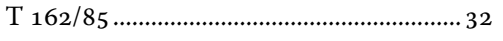 & ................... 40 \\
\hline
\end{tabular}




\begin{tabular}{|c|c|}
\hline $934 / 91 \ldots$. & $42 / 00 \ldots$ \\
\hline $341 / 92 \ldots \ldots . .$. & $48 / 00 \ldots . . . . .$. \\
\hline $374 / 92 \ldots \ldots$ & Г 641/oo......... \\
\hline $472 / 92 \ldots$. & $\mathrm{T} 950 / 00 \ldots . .$. \\
\hline $501 / 92 \ldots$ & T 1188/oo...... \\
\hline 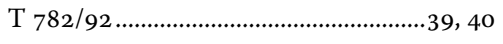 & T $55 / 01$ \\
\hline 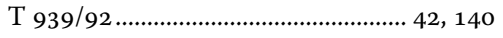 & 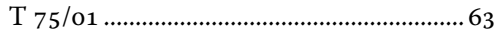 \\
\hline 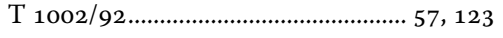 & 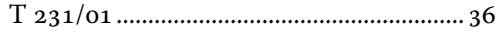 \\
\hline 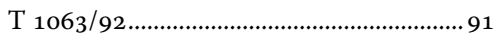 & T 520/01 \\
\hline 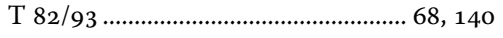 & $\mathrm{T} 681 / 01 \ldots . .$. \\
\hline $167 / 93 \ldots \ldots \ldots \ldots$ & Т 694/01 ...............................................161 \\
\hline $169 / 93 \ldots \ldots \ldots \ldots \ldots \ldots \ldots$ & $\mathrm{T} 713 / 01 \ldots \ldots \ldots \ldots . . . . .$. \\
\hline$\Gamma 292 / 93 \ldots \ldots \ldots \ldots \ldots \ldots \ldots \ldots$ & $\mathrm{T}$ 754/01 ............................................115 \\
\hline 793/93 …..................... & 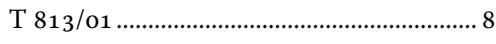 \\
\hline 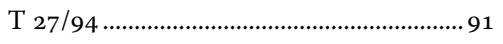 & $\mathrm{T} 1081 / 01 \ldots \ldots$ \\
\hline 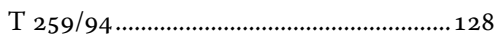 & $\mathrm{T} 1121 / 01 \ldots \ldots . . .$. \\
\hline$\Gamma$ 501/94............ & $\mathrm{T} 21 / 02 \ldots \ldots \ldots$ \\
\hline T 609/94.......................... & ............................. 35 \\
\hline T 227/95 ............. & 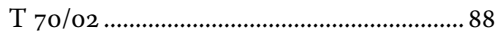 \\
\hline 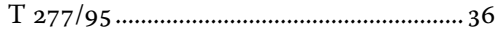 & .................... 98,99 \\
\hline Г $450 / 95 \ldots \ldots \ldots . . .$. & $\mathrm{T}_{389 / 02}$ \\
\hline T $817 / 95 \ldots . . .$. & $\mathrm{T} 643 / 02 \ldots$ \\
\hline T 919/95 ...... & $\mathrm{T} 723 / 02 \ldots \ldots$. \\
\hline $\mathrm{T}$ 37/96 ............. & $\ldots \ldots \ldots \ldots . .150$ \\
\hline T 142/96 ........................................155 & T 81/03 .................................115, 116 \\
\hline 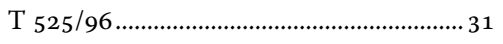 & T 281/03 \\
\hline 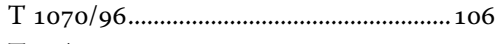 & T 602/03 ........................ \\
\hline $\mathrm{T} 83 / 97 \ldots \ldots \ldots . . .$. & T 910/03......... \\
\hline $\mathrm{T} 697 / 97 \ldots \ldots \ldots$. & $\mathrm{T} 1102 / 03 \ldots \ldots \ldots$ \\
\hline T $1067 / 97 \ldots \ldots . . . .$. & 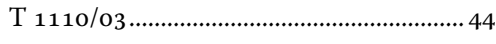 \\
\hline Т 1198/97 ........................ & $\mathrm{T} 1239 / 03 \ldots \ldots \ldots \ldots . . . .$. \\
\hline 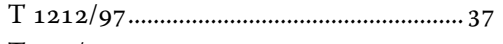 & 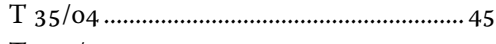 \\
\hline T 257/98 ............. & $\mathrm{T} 448 / 04 \ldots \ldots \ldots . . .$. \\
\hline $\mathrm{T} 473 / 98 \ldots \ldots \ldots \ldots$ & $\mathrm{T} 474 / 04 \ldots \ldots \ldots . .$. \\
\hline T $502 / 98 \ldots \ldots$. & $\mathrm{T} 763 / 04 \ldots \ldots \ldots$ \\
\hline $\mathrm{T} 962 / 98 \ldots \ldots \ldots \ldots \ldots$ & 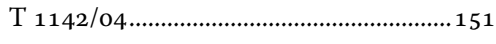 \\
\hline 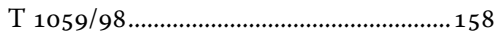 & T 1225/04 \\
\hline T 190/99...........................................6 63 & T 1415/04 ............................................... 71 \\
\hline 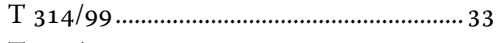 & 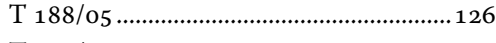 \\
\hline 40 & $\mathrm{~T} 222 / 05 \ldots \ldots \ldots$ \\
\hline 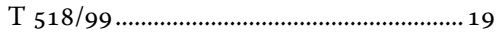 & $\ldots$ \\
\hline 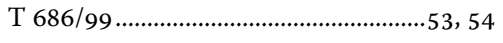 & 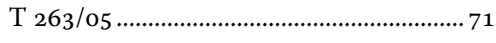 \\
\hline 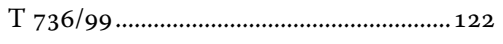 & 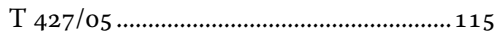 \\
\hline Г 1080/99 ...................................... 85,86 & T 482/05 ……............................................. 63 \\
\hline T $12 / 00 \ldots . . . . . . .$. & 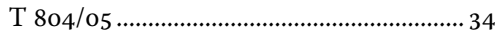 \\
\hline
\end{tabular}




\begin{tabular}{|c|c|}
\hline $906 / 05$. & $421 / 08 \ldots$ \\
\hline $922 / 05 \ldots$. & {$[491 / 08 \ldots$} \\
\hline $1119 / 05 \ldots .$. & $\Gamma 508 / 08 \ldots$. \\
\hline $1404 / 05 \ldots \ldots . . .$. & Г $541 / 08 \ldots \ldots . .$. \\
\hline $53 / 06 \ldots \ldots . . . .$. & Г $620 / 08 \ldots$. \\
\hline 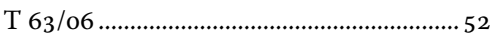 & Г 716/08 \\
\hline $204 / 06$ & $\mathrm{~T} 724 / 08$ \\
\hline 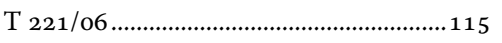 & T 1045/o8 \\
\hline $339 / 06 \ldots \ldots$. & 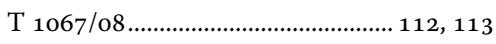 \\
\hline 910/06 ........... & Т $1126 / 08 \ldots$. \\
\hline 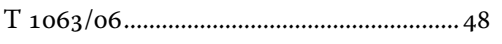 & T 1194/o8 \\
\hline 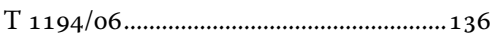 & T 1200/08 \\
\hline $1474 / 06 \ldots . . . . . .$. & T $1248 / 08 \ldots .$. \\
\hline Г 1553/06 & T 1488/o8 \\
\hline 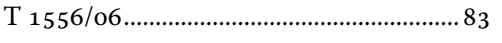 & T 1497/08............ \\
\hline 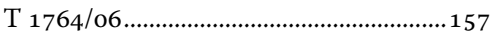 & 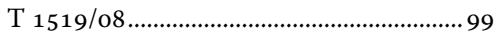 \\
\hline $1790 / 06 \ldots \ldots \ldots$ & . \\
\hline Г 1903/06........ & T $1810 / 08 \ldots \ldots$. \\
\hline 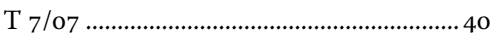 & T 1841/o8 \\
\hline Г 146/07 ……....................................92, 93 & 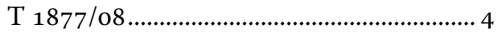 \\
\hline 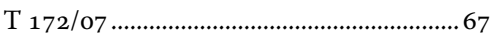 & T 1969/o8 ..........................................157 \\
\hline T $475 / 07 \ldots . .$. & $\mathrm{T} 2003 / 08 \ldots \ldots$ \\
\hline$\ldots \ldots \ldots \ldots \ldots \ldots \ldots . .55$ & $\mathrm{~T} 2006 / 08 \ldots \ldots \ldots$ \\
\hline 565/07 ……..................................115 & T 2165/o8 \\
\hline Г 608/07 …......................................49, 50 & 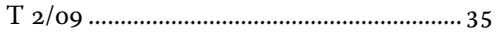 \\
\hline 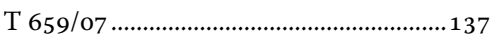 & T 67/09 .................. \\
\hline 50 & T $144 / 09 \ldots \ldots . . .$. \\
\hline 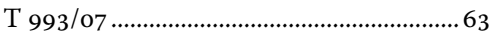 & 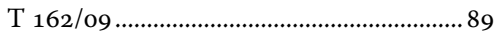 \\
\hline Г 1491/o7 ...........................................105 & $\mathrm{T} 183 / 09 . . . . . . . . .$. \\
\hline Г 1511/07 …....................................... 53 & T 226/09 …............................................. 99 \\
\hline Т 1587/07 & T 267/09 ..........................................117 \\
\hline 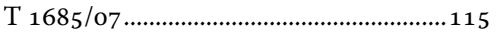 & T $278 / 09 \ldots \ldots$. \\
\hline Г $1705 / 07 \ldots \ldots$ & $\mathrm{T} 360 / 09 \ldots . . .$. \\
\hline 64 & $\mathrm{~T} 361 / 08 \ldots \ldots$. \\
\hline T 1870/07 ..................................... 88, 149 & 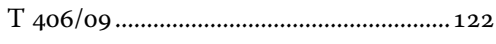 \\
\hline T 1990/07.........................................119 & 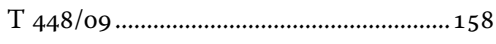 \\
\hline 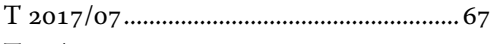 & $\mathrm{T} 459 / 09 \ldots \ldots$. \\
\hline 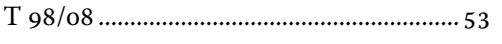 & $\mathrm{T} 473 / 09 \ldots . .$. \\
\hline $\mathrm{T} 177 / 08 \ldots \ldots . .$. & $\mathrm{T} 476 / 09 \ldots$ \\
\hline Г 232/08 ….........................................126 & 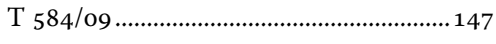 \\
\hline T 246/08 ........................................ 88, 150 & $\ldots$ \\
\hline 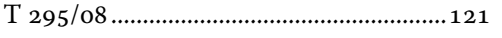 & 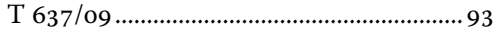 \\
\hline Т 316/08 & T 691/09 .........................................112 \\
\hline$\ldots \ldots \ldots \ldots . .137$ & $\mathrm{~T} 705 / 09 \ldots \ldots . .$. \\
\hline ..............112 & Г 834/09.... \\
\hline
\end{tabular}




\begin{tabular}{|c|c|}
\hline 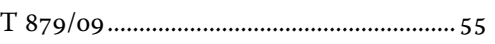 & 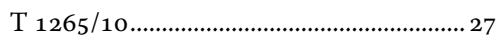 \\
\hline 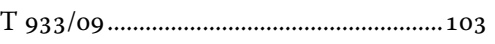 & 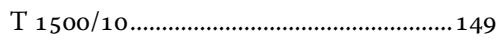 \\
\hline $\mathrm{T} 1111 / 09 \ldots \ldots$ & $\mathrm{T} 1525 / 10 \ldots \ldots \ldots$ \\
\hline T 1129/o9........... & $\mathrm{T} 1651 / 10 \ldots \ldots$ \\
\hline 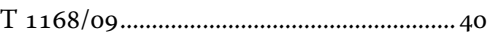 & $\mathrm{T} 1732 / 10 \ldots \ldots \ldots .$. \\
\hline Г $1361 / 09 \ldots \ldots$. & $\mathrm{T} 1761 / 10 \ldots \ldots \ldots$ \\
\hline T 1621/09.........................................62, 128 & $\mathrm{~T} 2165 / 10 \ldots \ldots .$. \\
\hline 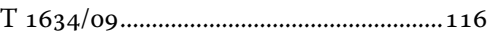 & $\mathrm{T} 2542 / 10 \ldots \ldots \ldots$ \\
\hline 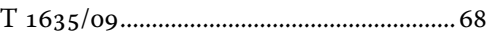 & $\mathrm{T} 134 / 11 \ldots \ldots \ldots \ldots$ \\
\hline T $1797 / 09 \ldots .$. & $\mathrm{T} 2532 / 11 \ldots$. \\
\hline 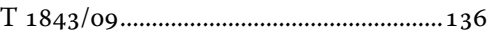 & $\mathrm{T} 383 / 11 \ldots \ldots \ldots \ldots$ \\
\hline T $1920 / 09 \ldots \ldots$. & $\mathrm{T} 480 / 11 \ldots \ldots .$. \\
\hline T 1959/09........ & $\mathrm{T} 553 / 11 \ldots \ldots . .$. \\
\hline T 1961/09......... & . 70 \\
\hline 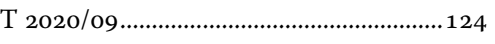 & 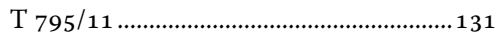 \\
\hline 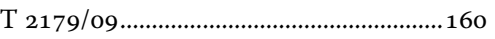 & T 880/11 \\
\hline T 2285/09............ & 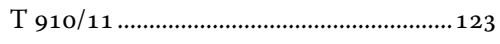 \\
\hline 33 & 127 \\
\hline T $2377 / 09 \ldots . .$. & $\mathrm{T} 1518 / 11 \ldots \ldots$ \\
\hline 149 & $\mathrm{~T} 1713 / 11 \ldots \ldots \ldots$ \\
\hline $\mathrm{T}_{5 / 10} \ldots \ldots \ldots . .$. & $\mathrm{T} 1843 / 11 \ldots \ldots$ \\
\hline .....................67 & ...................... 43, 137 \\
\hline ........ 104, 111 & 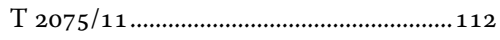 \\
\hline 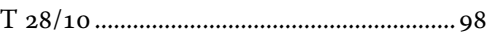 & 102 \\
\hline 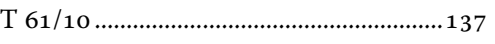 & T 2638/11 ...........................117, 118, 119 \\
\hline 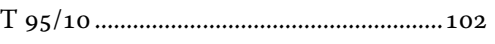 & 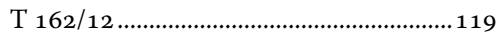 \\
\hline T 260/10 & T 373/12 \\
\hline $\mathrm{T} 351 / 10 \ldots \ldots . .$. & 102 \\
\hline T $358 / 10 \ldots \ldots . .150$ & 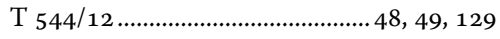 \\
\hline$\ldots \ldots \ldots \ldots \ldots \ldots . . .53$ & $\ldots \ldots \ldots \ldots \ldots \ldots . . .162$ \\
\hline 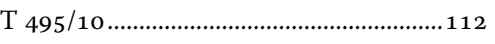 & T 2017/12 100 \\
\hline . & . \\
\hline 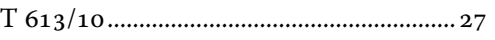 & T 1060/13 \\
\hline 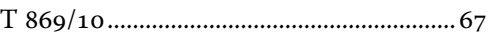 & 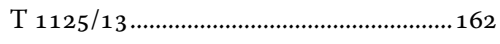 \\
\hline 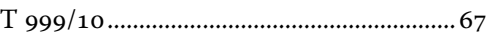 & 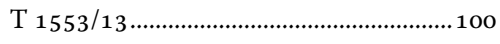 \\
\hline
\end{tabular}

